\title{
Tuberculose cutânea disseminada com escrofuloderma associado à tuberculose de arco costal ${ }^{*}$ Disseminated cutaneous tuberculosis with scrofuloderma associated to costal arch tuberculosis"
}

\author{
Elson Vidal Martins Junior ${ }^{1}$ \\ Bruno Pompeu Marques ${ }^{2}$ \\ Bianca Cristina M. L. de Souza Lima ${ }^{4} \quad$ Yuri Reinhardt B. Neumann ${ }^{5}$
}

Edgard Torres dos Reis Neto ${ }^{3}$

\begin{abstract}
Resumo: Os autores relatam caso de tuberculose cutânea disseminada com escrofuloderma associado à tuberculose de arco costal. Paciente de 46 anos, do sexo feminino, há um ano com nódulos de um a $6 \mathrm{~cm}$ em região cervical, dorso, axilas e regiões glúteas, que culminavam com fistulização e eliminação de secreção purulenta, associados a febre vespertina diária, sudorese noturna e emagrecimento de $10 \mathrm{~kg}$ nos últimos três meses. A radiografia de tórax mostrou lesão lítica na terceira costela esquerda. A cultura de secreção do nódulo foi positiva para Mycobacterium tuberculosis. O tratamento para tuberculose resultou em melhora clínica e resolução das lesões cutâneas da paciente.

Palavras-chave: Pele; Tuberculose; Tuberculose cutânea
\end{abstract}

\begin{abstract}
The authors describe a case of disseminated cutaneous tuberculosis with scrofuloderma associated to tuberculosis in the costal arch. A 46-year-old, woman, was bospitalized with nodules measuring 1 to $6 \mathrm{~cm}$ in the cervical region, back, armpit and buttocks, which developed purulent discharge with elimination of caseous secretion, associated to episodes of daily evening fever, night sweats and weight loss of $10 \mathrm{Kg}$ over the previous three months. Thorax radiography showed an osteolytic lesion in the third left rib. Culture of the nodule secretion was positive for Mycobacterium tuberculosis. Treatment for tuberculosis resulted in pronounced clinical improvement and resolution of the patient's cutaneous lesions.
\end{abstract}

Keywords: Cutaneous tuberculosis; Skin; Tuberculosis

\footnotetext{
Recebido em 26.04.2005.

Aprovado pelo Conselho Consultivo e aceito para publicação em 30.05.2007.

* Trabalho realizado no Serviço de Clínica Médica do Hospital Guilherme Álvaro da Faculdade de Ciências Médicas de Santos - Santos (SP), Brasil. Conflito de interesse: Nenhum / Conflict of interest: None

Suporte financeiro: Nenhum / Financial funding: None

Professor de Clínica Médica da Faculdade de Ciências Médicas de Santos. Mestre em Gastroenterologia pela Universidade Federal de São Paulo (Unifesp) - São Paulo (SP), Brasil.

Professor de Clínica Médica da Faculdade de Ciências Médicas de Santos - Santos (SP), Brasil.

Residente de Clínica Médica do Hospital Guilherme Álvaro de Santos - São Paulo (SP), Brasil.

Residente de Clínica Médica do Hospital Guilherme Álvaro de Santos - São Paulo (SP), Brasil.

Estudante de Medicina da Faculdade de Ciências Médicas de Santos - Santos (SP), Brasil.

C) 2007 by Anais Brasileiros de Dermatologia
} 


\section{INTRODUÇÃO}

A tuberculose é infecção causada pelo Mycobacterium tuberculosis, também conhecido como bacilo de Koch (BK). O Brasil, juntamente com outros 21 países em desenvolvimento, alberga 80\% dos casos mundiais da doença. Um terço da população mundial está infectada, permanecendo sob risco de desenvolver a enfermidade. No Brasil, estima-se que mais de 50 milhões de pessoas estejam infectadas, com aproximadamente 100 mil casos novos por ano. Com o advento da síndrome de imunodeficiência adquirida, a resistência bacteriana a drogas antituberculosas e o uso mais freqüente de imunossupressores, vêm-se observando crescente número de casos notificados da doença e aumento de sua morbimortalidade. ${ }^{1,2}$

A tuberculose comumente se localiza nos pulmões, embora possa acometer qualquer órgão, sendo então denominada tuberculose extrapulmonar; pode afetar os gânglios linfáticos, a medula óssea, a pele, o fígado, as meninges, o trato genitourinário e gastrointestinal, o sistema osteoarticular, o peritônio, entre outros. $^{3}$

O escrofuloderma é forma de tuberculose cutânea, também denominada escrófula ou tuberculose gomosa, que atinge o tecido subcutâneo e provoca fistulização e ulceração da pele que o recobre. ${ }^{4,5}$ Predomina em crianças e adultos jovens. O mecanismo de infecção é endógeno, ou seja, provém de focos tuberculosos prévios que invadem a pele por contigüidade, geralmente com origem em lesões ósseas, articulares ou nos gânglios linfáticos, o que explica na maioria dos casos a topografia das lesões. ${ }^{5}$

O tratamento consiste no uso de drogas antituberculosas. A maioria dos pacientes responde efetivamente às medicações utilizadas no tratamento da tuberculose pulmonar. ${ }^{5}$

O objetivo do artigo é relatar caso de tuberculose cutânea disseminada com escrofuloderma associado à tuberculose de arco costal e realizar revisão de literatura acerca dessa rara ocorrência.

\section{RELATO DO CASO}

Paciente do sexo feminino, de 46 anos de idade, foi admitida no Hospital Guilherme Álvaro (Santos/SP) em maio de 2004 com nódulos de consistência amolecida, indolores, com rubor e calor local, não aderidos a planos profundos, localizados na região cervical, dorso, axilas e região glútea; mediam de um a $6 \mathrm{~cm}$ de diâmetro, com aumento progressivo de tamanho, culminando com fistulização e eliminação de secreção purulenta. À lesão associavam-se febre vespertina diária com calafrios e sudorese noturna há um ano, e emagrecimento de $10 \mathrm{~kg}$ nos últimos três meses. Histórico de irmão com tratamen- to para tuberculose há dois anos. Ao exame físico apresentava-se em regular estado geral, caquética e hipocorada $(3+/ 4+)$. Notava-se a presença de cinco nódulos na região esternal e supraclavicular esquerda e direita, de $3 \mathrm{~cm}$ de diâmetro, indolores, com rubor, de consistência amolecida, móveis, sendo três ulcerados, com saída de secreção purulenta e dois em fase de cicatrização. Acrescentem-se dois nódulos de $6 \mathrm{~cm}$ de diâmetro e com ulceração, no dorso, e um na região axilar direita e no flanco direito, de iguais características (Figuras 1A e B). Exames laboratoriais mostraram hemoglobina de $5,8 \mathrm{~g} / \mathrm{dl}$ com hipocromia e microcitose; leucometria com 7.650 células $/ \mathrm{mm}^{3} \mathrm{com}$ contagem diferencial normal; velocidade de hemossedimentação de $90 \mathrm{~mm} / 1^{\mathrm{a}}$ hora; proteínas totais de $8 \mathrm{mg} / \mathrm{dl}$ e albumina igual a $1,8 \mathrm{mg} / \mathrm{dl}$. As sorologias anti-HIV e VDRL foram não reagentes; o PPD foi não reator, e a baciloscopia no escarro, negativa (três amostras).

O cultivo de material obtido por punção aspirativa do nódulo localizado no dorso foi positivo para Mycobacterium tuberculosis. A biópsia do nódulo revelou a presença de granuloma de corpo estranho, tecido de granulação e microcalcificação, e detectou bacilos à coloração de Ziehl-Neelsen.

A radiografia de tórax (Figura 2) revelou lesão lítica na terceira costela esquerda, sem evidência de tuberculose pulmonar. A tomografia computadorizada de tórax mostrou linfonodomegalias no espaço pré-vascular e espessamento pleural no ápice e terço médio posterior esquerdo. A cintilografia óssea de corpo total apontou captação aumentada no terceiro arco costal esquerdo.

Instituiu-se o esquema I (rifampicina, isoniazida e pirazinamida) para tratamento de tuberculose, que foi, porém, suspenso por hepatotoxicidade revelada por elevação das eñ̇ imas hepáticas e aumento das bilirrubinas (AST: 226U/L, ALT: 222U/L, bilirrubina total: $2,57 \mathrm{mg} / \mathrm{dl}$ e bilirrubina direta: $1,97 \mathrm{mg} / \mathrm{dl}$ ), sendo introduzido esquer na modificado (etambutol, estreptomicina e isoniazida) após normalização laboratorial. A paciente evoluiu afebril, com melhora progressiva do estado geral e nutricional, apresentando regressão e cicatrização $d$ is lesões (Figuras 3A e B), recebendo alta hospitalı́ $\mathbf{r}$ para acompanhamento ambulatorial.

\section{DISCUSSÃO}

A lesão elementar d s escrofuloderma, denominada goma ou escrófulo, jode ser única ou múltipla e evolui em cinco etapas: enduração, amolecimento, fistulização, ulceração e cicatrização, podendo ser observados todos os estágios da lesão em um mesmo paciente. ${ }^{5}$ Inicialmente os escrófulos apresentam-se 

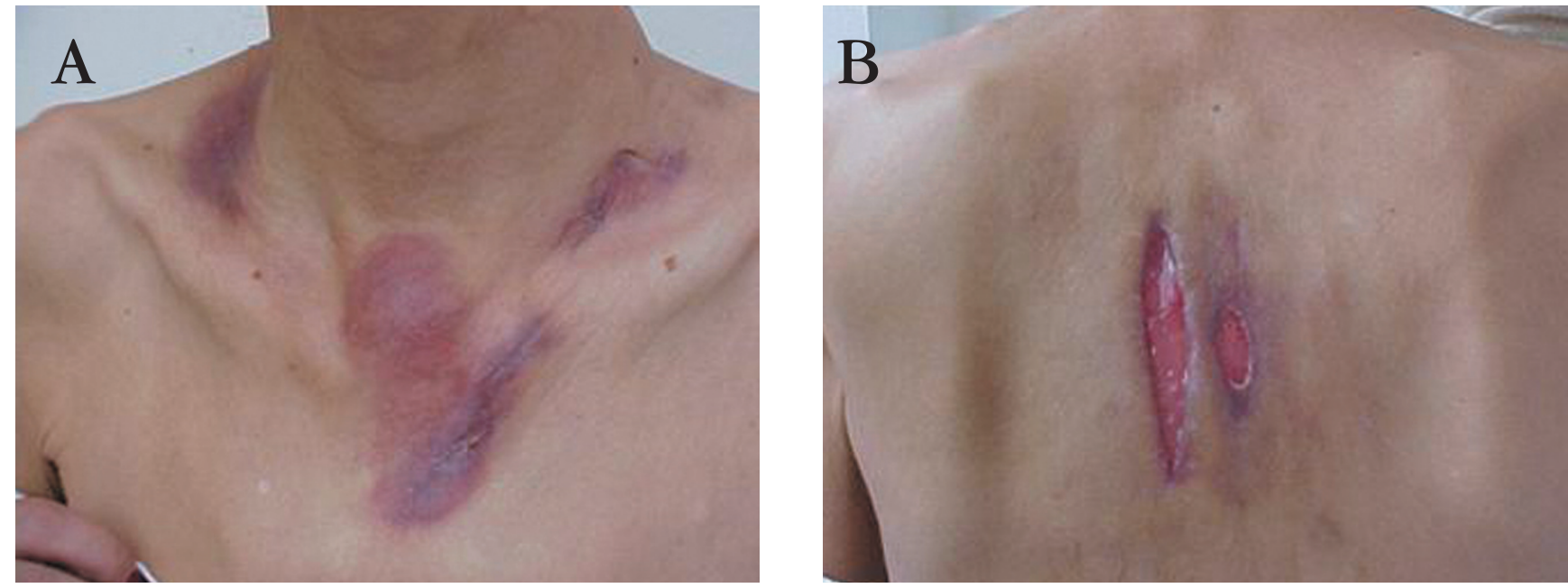

Figuras 1 A E B: Lesões iniciais da tuberculose cutânea apresentada pela paciente

como nódulos subcutâneos, endurecidos e móveis, que se tornam pastosos, com posterior flutuação e perfuração da pele que os recobre, formando úlceras e fístulas que se comunicam e drenam material aquoso, purulento ou caseoso.

Trata-se de doença rara, cuja exata prevalência e incidência são desconhecidas. Em estudo realizado na Turquia em 370 pacientes com tuberculose visceral, evidenciaram-se oito pacientes com escrofuloderma com lesão na região cervical $(2,16 \%)$, com média de idade de 24,8 anos (variando de 11 a 51 anos), sendo $50 \%$ de cada sexo. Deles, quatro casos apresentavam tuberculose pulmonar ativa, três apresenta-

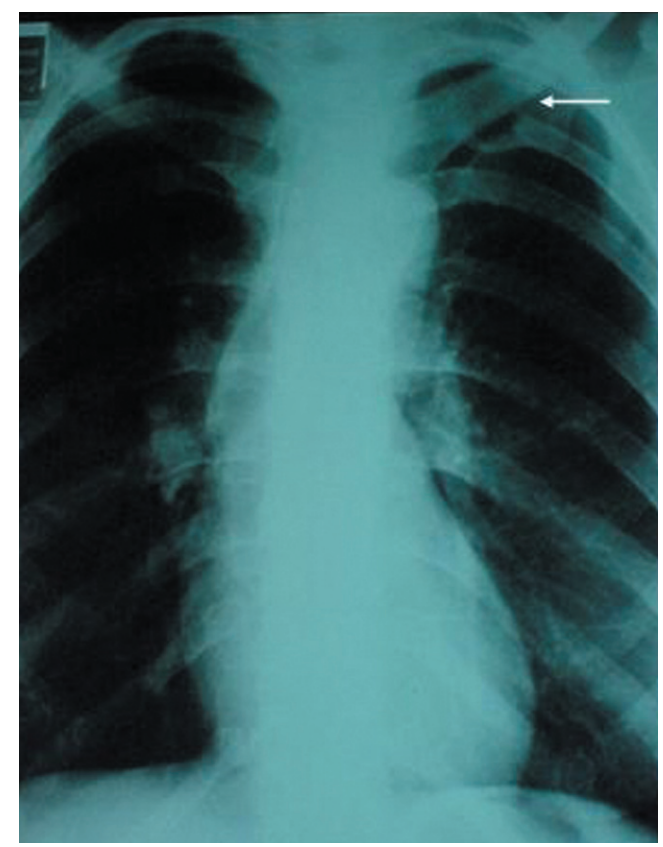

Figura 2: Radiografia de tórax com lesão lítica em terceira costela esquerda vam tuberculose ganglionar, e um. tuberculose pleuropulmonar. Cinco pacientes tinham história familiar positiva para tuberculose. O escrofuloderma e o lúpus vulgar foram as formas clínicas mais freqüentes de tuberculose cutânea observadas. ${ }^{2} \mathrm{Na}$ Tailândia, entre 104 pacientes com tuberculose cutânea e infecções cutâneas micobacterianas não tuberculosas diagnosticadas, detectaram-se 18 pacientes com escrofuloderma, com média de idade de 36,9 anos (variando de 11 a 61 anos) e 50\% de cada sexo; 16 apresentavam linfadenite como foco subjacente -15 na região cervical, e um na região inguinal -, sendo que um caso tinha múltiplos abscessos frios nos membros superiores e inferiores, com drenagem. Nesse trabalho, o agente etiológico foi isolado em 14 casos (77,7\%), sendo seis decorrentes do Mycobacterium tuberculosis, e oito de outras micobactérias. ${ }^{6}$ Em nenhum desses estudos há relato de escrofuloderma associado à tuberculose óssea, o que, porém, já é bem-estabelecido na literatura. ${ }^{3,5,7-10}$ Entretanto, escrofuloderma associado à tuberculose de arco costal constitui evento raro, sendo do conhecimento dos autores apenas um relato de caso em criança. ${ }^{8}$

A tuberculose óssea ou articular constitui aproximadamente $10 \%$ de todas as formas de tuberculose extrapulmonar. ${ }^{8}$ A localização em arco costal ocorre em percentual que varia de um a $3 \%$ dos casos, sendo geralmente subdiagnosticada. ${ }^{8,10} \mathrm{~A}$ infecção atinge os ossos por disseminação linfática e/ou hematogênica, embora a infecção por contigüidade de linfonodos também ocorra. ${ }^{8}$

As lesões líticas nas costelas são usualmente de origem neoplásica ou infecciosa, sendo os homens duas vezes mais acometidos que as mulheres, e a idade mais comum entre 15 e 35 anos. ${ }^{7}$ O sarcoma de Ewing, o fibrossarcoma, o mieloma múltiplo, a 

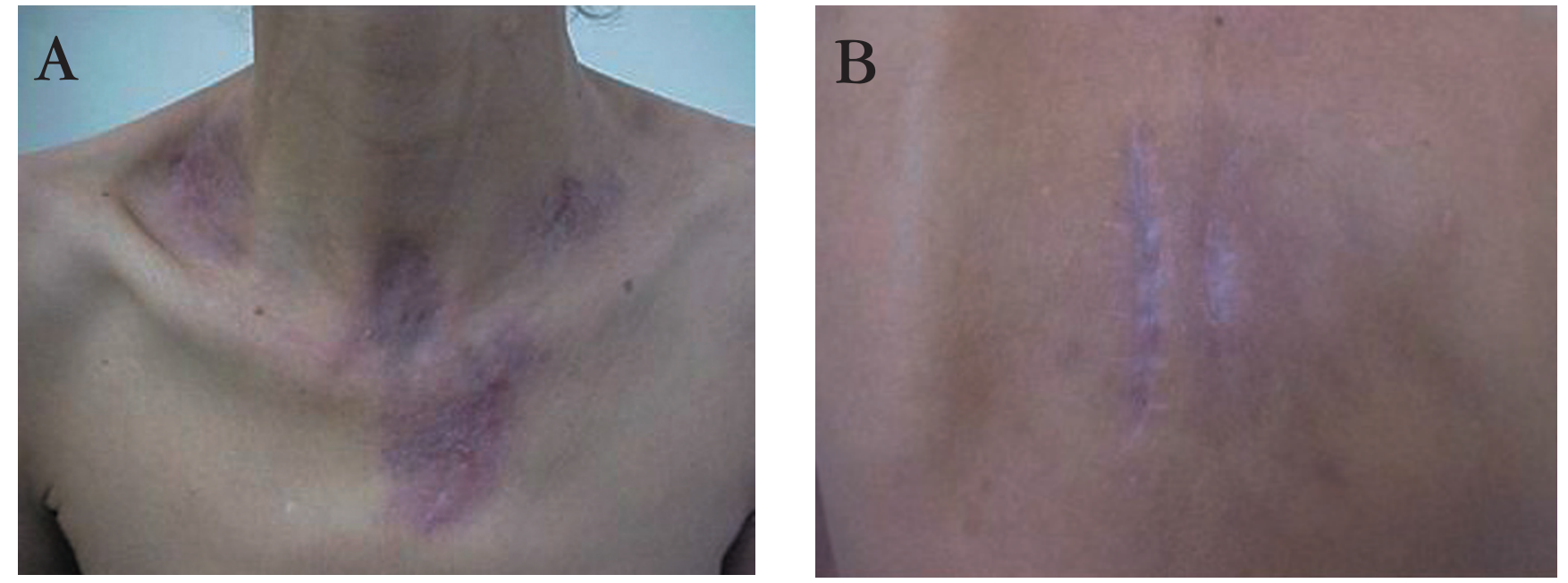

Figuras 3 A E B: Regressão e cicatrização das lesões após terapêutica instituída

histiocitose; bem como metástases de mama, fígado, tireóide e rim produzem lesões osteolíticas semelhantes às conseqüentes a doenças infecciosas. ${ }^{8}$ Após as doenças neoplásicas, a tuberculose é a segunda causa mais comum de lesões destrutivas na costela. ${ }^{7}$ Estudos sugerem que o foco de tuberculose localizado na periferia dos pulmóes possa disseminar-se diretamente através da pleura até a superfície visceral da costela ou que um empiema possa, per se, iniciar processo inflamatório na superfície da costela. ${ }^{9}$ A lesão de arco costal pode manifestar-se como dor torácica, massa na parede torácica ou por fistulização.

A radiografia de tórax pode não detectar lesão óssea na fase inicial, atrasando o diagnóstico. ${ }^{7,10}$ As alterações possíveis incluem processo expansivo ou espessamento, erosões e, mais raramente, formação de seqüestro ósseo. ${ }^{10}$ A tomografia computadorizada de tórax pode mostrar abscesso de tecidos moles, lesões osteolíticas, seqüestro ou, menos comumente, esclerose óssea. ${ }^{11}$ No caso em questão, a cintilografia óssea mostrou hiperconcentração apenas no terceiro arco costal esquerdo, afastando lesão tuberculosa óssea em outros locais.

$\mathrm{O}$ achado histopatológico da área central da lesão cutânea mostrou necrose central maciça com formação de abscesso. Nos bordos da úlcera e nas margens da fístula podem-se observar granulomas tuberculóides com necrose de caseificação e a presença do Mycobacterium tuberculosis. ${ }^{4,12}$ O granuloma da tuberculose é formado por uma ou mais células gigantes, geralmente do tipo Langhans, ao redor das quais existem várias células epitelióides. Dois ou mais desses granulomas podem fundir-se, formando nódulos visíveis (tubérculos miliares). Na periferia, há numerosos linfócitos, alguns macrófagos e poucos plasmócitos. ${ }^{12}$

Em todo paciente deve-se buscar algum foco de tuberculose subjacente. ${ }^{3} \mathrm{O}$ diagnóstico diferencial deve ser feito com sífilis, esporotricose ${ }^{4,5}$ abscessos frios, actinomicose cervicofacial, paracococcidioidomicose, linfoma de Hodgkin, ${ }^{5}$ acne conglobrata e hidradenite supurativa. ${ }^{4}$ Das complicações são referidas a infecção secundária e a formação de cicatrizes profundas de aspecto rugoso. ${ }^{5}$ Há ainda relato de casos da síndrome de Sweet em pacientes em tratamento para escrofuloderma. ${ }^{6}$

O tratamento consiste no uso de drogas antituberculosas, sendo indicado no Brasil, segundo orientação atual do Ministério da Saúde, o esquema inicial de pirazinamida por dois meses e rifampicina e isoniazida por seis meses. ${ }^{1}$ Além disso, deve-se melhorar o estado nutricional do paciente, bem como tratar infecções coexistentes ou intercorrentes. Após anos de evolução sem tratamento, pode ocorrer a cura espontânea, quando as lesões inflamatórias são substituídas por tecido cicatricial característico com aspecto de quelóide "em cordão". ${ }^{4}$

O tratamento cirúrgico com retirada das lesões do escrofuloderma pode ser considerado ${ }^{4,8}$ A cirurgia da lesão óssea pode ser necessária para confirmação diagnóstica em casos mais complicados, em lesões avançadas com caseificação, quando há destruição óssea ou fibrose com cicatrização e visando à remoção de grande área de seqüestro ósseo, e nos casos não responsivos ao tratamento farmacológico. ${ }^{10} \mathrm{No}$ presente caso, observou-se excelente resposta com o tratamento clínico. 


\section{REFERÊNCIAS}

1. Brasil. Ministério da Saúde. Guia de Vigilância Epidemiológica. Brasília: Ministério da Saúde; 2003. p.824-46.

2. Kinvanç-Altunay I, Baysal Z, Ekmekçi TR, Köslu A Incidence of cutaneous tuberculosis in patients with organ tuberculosis. Int J Dermatol. 2003;42:197-200.

3. Domínguez O, Guillén M. Tuberculosis. El médico. 2000;19:25-52

4. Paez TC, Palomero TSL, Mota ED, Serrano SC. Tuberculosis ganglionar. Medicina General. 2001;35:529-32.

5. Rodríguez O. Tuberculosis cutánea. Rev Fac Med UNAM. 2003;46:157-61.

6. Mahaisavariya P, Chaiprasert A, Manonukul J, Khemngern S. Scrofuloderma and Sweet's syndrome. Int J Dermatol. 2002;41:28-31.

7. Asnis DS, Niegowska A. Tuberculosis of the rib. Clin Infect Dis. 1997;24:1018-9.

8. Kaur S, Thami GP, Gupta PN, Kanwar AJ. Recalcitrant scrofuloderma due to rib tuberculosis. Pediatr Dermatol. 2003;20:309-12.
9. Roberts C, Lucy D, Manchester K. Inflammatory lesions of the ribs: an analysis of Terry collection. Am J Phys Anthropol.1994;95:169-82.

10. Tuli SM. Tuberculosis of skeletal system. New Delhi: Jaypee; 1991. p.121-2.

11. Khalil A, La Breton C, Tassart M, Korzec J, Bigot J Carette M. Utility of CT scan for the diagnosis of chest wall tuberculosis. Eur J Radiol. 1999;9:1638-42.

12. Cotran RS, Robbins SL, Kumar V. Fundamentos de patologia estrutural e funcional. Robbins SL, Cotran RS. Eds. 6 ed. Rio de Janeiro: Guanabara Koogan; 2000. p.314-6.

ENDEREÇO PARA CORRESPONDÊNCIA / MAILING ADDRESS: Elson Vidal Martins Júnior Av. Epitácio Pessoa 68/62 Santos - SP 11045-300 - Santos - SP E-mail: elson.vidal@terra.com.br; edgardtorres@terra.com.br

Como citar este artigo: Martins Junior EV, Marques BP, Reis Neto ET, Lima BCMLS, Neumann YRB. Tuberculose cutânea disseminada com escrofuloderma associado à tuberculose de arco costal. An Bras Dermatol. 2007;82(4):343-47.

How to cite this article: Martins Junior EV, Marques BP, Reis Neto ET, Lima BCMLS, Neumann YRB. Disseminated cutaneous tuberculosis with scrofuloderma associated to costal arch tuberculosis. An Bras Dermatol. 2007;82(4):343-47. 